\title{
Impacto de las tecnologías disruptivas en el proceso de enseñanza - aprendizaje: caso UTM online
}

Fecha de recepción: 2021-09-27 • Fecha de aceptación: 2021-11-01 • Fecha de publicación: 2022-01-10

\author{
Walter José Zambrano Romero' \\ Universidad Técnica de Manabí, Ecuador \\ jose.zambrano@utm.edu.ec \\ https://orcid.org/0000-0001-9747-4808 \\ Jaime Alcides Meza Hormaza² \\ Universidad Técnica de Manabí, Ecuador \\ jaime.meza@utm.edu.ec \\ https://orcid.org/0000-0002-8279-5630
}

\section{Resumen}

En la actualidad los entornos educativos en línea mediados por tecnologías han desarrollado ambientes de innovación en los procesos pedagógicos que facilitan el aprendizaje de los educandos, la presente investigación tiene como objeto de medir el impacto que tiene el uso de las tecnologías disruptivas en el proceso de enseñanza y aprendizaje de las carreras en línea de UTM online. Con el propósito de integrar estas innovaciones a los centros de Educación Superior, y de proveer nuevos mecanismos de construcción de significados. En cuanto al estudio, parte de una intencionalidad exploratoria, el enfoque utilizado en esta investigación fue de corte cuantitativo con un diseño no experimental y transversal, de alcance descriptivo, realizando un estudio del fenómeno comparado con otras asignaturas. Por otra parte, en los resultados se observa que el $80 \%$ de los participantes dieron una calificación correspondiente a totalmente satisfactorio. Lo que demuestra que los participantes perciben un mejor aprendizaje al implementar aplicaciones digitales y plataformas colaborativas en el desarrollo de prácticas en tiempo real discrepando inquietudes. La investigación sugiere que las herramientas digitales como Google Docs, Quizizz y Flipgrid son herramientas de 
aprendizaje útiles en su adaptación para fomentar la interacción y la retención del aprendizaje en línea.

Palabras clave: educación en línea, tecnologías disruptivas, enseñanza y aprendizaje, plataformas colaborativas

\begin{abstract}
Currently, online educational environments mediated by technologies have developed innovation environments in pedagogical processes that facilitate student learning, the present research aims to measure the impact of the use of disruptive technologies in the process of learning. Teaching and learning of online UTM careers online. In order to integrate these innovations to Higher Education centers, and to provide new mechanisms for the construction of meanings. As for the study, it starts from an exploratory and descriptive intention of knowing in depth the problem in the teaching and learning process in educational practice, where the quantitative methodology of a non-experimental nature was applied. On the other hand, in the results, it is observed that $80 \%$ of the participants gave a corresponding rating to very satisfactory. This shows that the participants perceive better learning when implementing digital applications and collaborative platforms in the development of real-time practices, disagreeing with concerns. Research suggests that digital tools like Google Docs, Quizizz, and Flipgrid are useful learning tools in their adaptation to encourage interaction and retention of online learning.
\end{abstract}

Keywords: online education, disruptive technologies, teaching and learning, collaborative platforms 


\section{Introducción}

Actualmente, la formación académica a nivel de Latinoamérica y especialmente la educación superior, se ven compulsadas al encontrar metodologías y estrategias didácticas en potenciar la enseñanza y en la educación en línea (Pilonieta, 2017). Por tanto, se ha conducido en fortalecer el proceso de enseñanza y aprendizaje, utilizando tecnologías disruptivas con plataformas colaborativas, como medio para la introducción de contenidos interactivos e innovadores en la formación educativa.

Una de las instituciones de educación superior que adoptó estos procesos tecnológicos disruptivos fue la Universidad de Stanford en octubre de 2011, quienes utilizaron la realidad aumentada, la inteligencia artificial y el aprendizaje automático. El experimento fue catalogado como exitoso, captando la atención de más de cien mil personas en todo el mundo. Con estos resultados, los promotores de la idea y las universidades que los apoyaban decidieron crear programas mucho más completos y estructurados para hacer que los conocimientos de última generación pudieran llegar a un número mayor de individuos (Acevedo, 2015).

Al presente, no se trata de solo aprender el manejo de la tecnología, sino, de crear innovaciones en solventar las necesidades de las personas vulneradas como es la impresión 3D, que permite imprimir desde prótesis hasta accesorios de vehículos a bajo costo; sin embargo, lo que ha revolucionado en las tecnologías disruptivas es la inteligencia artificial, donde ha generado grandes avances que se observan en las compañías internacionales en sus entornos digitales, convirtiéndose en un asistente virtual con motricidad cognitiva del ser humano.

Las condiciones del entorno del último lustro han impulsado a las universidades a desarrollar y brindar una educación de calidad a los estudiantes, con un entorno de aprendizaje completo para los alumnos (Arriassecq \& Santos, 2017). Sin embargo, las universidades se enfrentan a los desafíos como son: los precios crecientes y la presión para brindar a los estudiantes el conocimiento apropiado, de crear a personas competitivas, con habilidades innovadoras y con la capacidad para adaptarse a los entornos laborales que están en constante cambios (Banoy Suarez, 2019).

En este sentido, la Universidad Técnica de Manabí (UTM) ha iniciado la adecuación de sus procesos al desarrollo de nuevas estrategias pedagógicas, incorporando tecnologías disruptivas para fortalecer la docencia en las carreras en línea. En este contexto, el objetivo de este estudio es medir el impacto que tiene el uso de las tecnologías disruptivas en el proceso de enseñanza y aprendizaje de UTM online, con el fin de establecer una línea base que permita que los profesores tutores adquieran características intrínsecas de orientar y guiar a sus alumnos como eje principal de su enseñanza, permitiendo que el aprendizaje mediado por tecnología provee a un aprendizaje activo y colaborativo.

\subsection{Herramientas disruptivas}

Las universidades como centro de estudio están sujetas a las dinámicas educativas, impulsando entre sus labores fundamentales la producción de conocimientos, por lo cual la formación y 
preparación de sus estudiantes al servicio de una sociedad más competitiva y desarrollada es una meta impostergable. En los establecimientos universitario, los procesos de enseñanza deberán estar ajustados a las innovaciones tecnológicas, de forma tal que tanto el personal académico, como docente, esté capacitado y actualizado en las Tecnologías de la Información y la Comunicación (TIC), de esta manera, las instituciones educativas, según sus particularidades culturales, económicas, tecnológicas, están asumido reformas educativas para su integración a las nuevas tecnologías disruptivas (Cabecero, 2010).

Las tecnologías disruptivas son aquellas que alteran las prácticas establecidas, comenzando con un número reducido de usuarios, pero creciendo con el tiempo hasta el punto de desplazar a una tecnología anterior dominante. Por el contrario, las tecnologías emergentes son tecnologías que mejoran el rendimiento de las ya establecidas (Flavin, 2012). Como señala Christensen \& Armstrong (1998), que todas las tecnologías emergentes tienen en común de mejorar el rendimiento de los productos establecidos, las tecnologías disruptivas aportan al mercado con un valor muy diferente al propuesto, los productos disruptivos están basados en tecnologías que suelen ser más económicas, en menor tamaño y fácil para implementar en cualquier entorno, ya sea de negocios o educativos.

Como plantea Flavin (2016), la disrupción no funciona al confrontar una práctica establecida, pero haciendo algo nuevo despierta la curiosidad de las personas por querer aprender algo novedoso: una lección importante de nuestros estudios de la improvisación es que la innovación disruptiva no se arraiga a través de un ataque directo en el sistema existente; es decir, debe rodear y por debajo del sistema. Por tanto, aplicar tecnologías digitales al proceso de enseñanza y aprendizaje intrínsecamente en los modelos pedagógicos establecidos, se espera que las tecnologías cambien el aprendizaje y la práctica. Por ende, el docente genera una problemática, porque las tecnologías tienden a deformarse para adaptarse a la pedagogía existente, de esta manera, el potencial disruptivo y transformador de las tecnologías no se realizan de inmediato lleva su proceso en adaptarse.

La tecnología y la innovación disruptiva se basan en su creciente popularidad de herramientas colaborativas, en la que se tienen preparaciones que desembocan a la innovación, desplegando nuevas estrategias de aprendizaje para estimular la capacidad en los alumnos, permitiendo mejorar su formación académica. Por ello, insertar tecnología como medio estratégico permite mejorar la interacción con el estudiante en las clases sincrónicas, generando debates y participando de manera crítica en la resolución de problemas, despertando su motivación por investigar y leer. Es importante destacar la empresa Google, que es un ejemplo de innovación disruptiva que está en constante cambio. Sin embargo, los proyectos de Google son desarrollados en base a las necesidades de los usuarios, con su equipo de ingenieros en sistemas y diseñadores que suministran a la plataforma con nuevas ideas y aplicaciones facilitando los requerimientos de los consumidores.

De acuerdo con Flavin \& Quintero (2018), la empresa Google se ha expandido en crear nuevos productos y servicios que ofrecen una gama de herramientas, desde académicos especializados búsquedas para documentar la composición y el almacenamiento, y así desarrollar su distribución inicial la innovación disruptiva a lo largo de líneas de innovación sustentable. 
Es por ello, que en el contexto y las circunstancias en las que se enmarca la educación y los instrumentos colaborativos, fenómenos cuya consagración ya es una realidad tras una etapa inicial de despegue. Este trabajo gira en torno a la obra de Clayton Christensen, considerado el "padre" de la innovación disruptiva, profesor de la afamada Escuela de Negocios de Harvard y un asiduo colaborador de la prestigiosa revista "Harvard Business Review". Entre sus aportaciones más destacadas, ha de reseñarse que fue quien acuñó el término o definición de innovación disruptiva, y marcó las guías para futuros análisis prácticos. Este análisis se encuentra cimentado sobre dos peculiaridades que han de reunir todos aquellos productos innovadores que quieran verse adjetivados por el término disruptivo. Finalmente, la obra de Christensen será recordada por "haber puesto a la innovación disruptiva en el mapa" (Diaz-leante, 2019).

En cuanto a la dimensión disruptiva de los Massive Open Online Course (MOOC) radica en la explotación de las tecnologías digitales para aumentar exponencialmente la velocidad a la que se crea y distribuye el conocimiento, reduciendo simultáneamente las barreras para crearlo y consumirlo. Sin embargo, no se puede suponer que el acceso a las tecnologías digitales sea equitativo y universal en el mundo en desarrollo, donde los diferentes niveles de exclusión social con respecto a Internet la conectividad persiste (McAuley et al., 2010).

\subsection{Educación disruptiva mediada por tecnología}

Los cambios disruptivos provocados por la educación, la tecnología y otras fuerzas están en el radar de analistas de educación, que incluso al indagar en el Internet se revela una gran cantidad de artículos y organizaciones sin fines de lucro, que están enfocadas en la innovación educativa (Kurtz et al., 2019). En pocas palabras, con los cambios disruptivos por tecnologías, algunos docentes generan conflictos con los procesos tecnológicos, que no desean actualizar su método tradicional de impartir la enseñanza, sino que se mantienen en el camino, su modelo tradicional, donde se torna con dificultad aplicar metodologías activas y de no insertar tecnologías disruptivas en el aula virtual.

Es indiscutible que los modelos tradicionales de educación no han desaparecido ni juzgamos que lo vayan a hacer en un tiempo intermedio. Por ende, los niños, adolescentes y jóvenes no asimilan el estudio como antes lo hacían, al momento de obtener el conocimiento que se está en constante cambio, se deberían hacer lo propio de innovar en la enseñanza. "No resulta una alteración, un cambio, de primer orden, el aprendizaje que hoy se hace, también desde instituciones presenciales, a través de plataformas digitales", el aprendizaje digital es totalmente diferente al presencial, sostiene García (2017).

El desarrollo de la educación superior está lidiando con el potencial disruptivo de las soluciones tecnológicas para mejorar la educación de manera rentable, sin poner en riesgo la formación del estudiante. Los desafíos impactan, tanto en los entornos académicos, como presenciales y virtuales, se exige una resiliencia y agilidad como algo crucial para el cambio. Las universidades que se esfuerzan por mantenerse al día con el ritmo del cambio, los roles transitorios y los procesos pedagógicos imperativos. Es decir, el aprendizaje se está centrado en el estudiante, donde se exploran estrategias eficientes que puedan minimizar los factores de riesgo (Hutchings \& Quinney, 2015)the propensity for student identities to shift from partners in learning to consumers of 
education provides challenges for negotiating the learning experience. Higher education institutions (HEls.

Por otra parte, al implementar las pedagogías disruptivas y tecnologías emergentes, estas son asociadas con "el aula invertida", que dan un aprendizaje transformador. De esta manera, se reconoce la importancia del aprendizaje colaborativo de generar cambios estratégicos, como requisitos previos en los procesos de mantener el cambio educativo. Según Gallardo et al. (2020), hay diferentes contribuciones que muestran el uso de dispositivos tecnológicos digitales, influyendo de forma determinante en el proceso de enseñanza aprendizaje, exponiendo su uso a una mejora significativamente con el proceso de adquisición de nuevos conocimientos, en lo que revierte positivamente el rendimiento académico más deseable y beneficioso.

Es por ello que se debe innovar e implementar nuevas metodologías activas en la pedagogía virtual, insertando en la educación en línea tecnologías disruptivas con plataformas colaborativas, que el estudiante observe, analice, realice preguntas para discernir sus inquietudes, generando debates con sus compañeros en búsqueda de la resolución de problemas y que practique en clases en línea, resolviendo algún ejercicio de un caso práctico. Es decir, el docente propone y cambia su roll a tutor, de guiar al estudiante generando parámetros claros en las actividades, donde el alumno analiza, resuelve el contenido de manera colaborativa, de que todos sus compañeros aporten de manera significativa, con la finalidad de que construyan su oportuno aprendizaje.

Al respecto, Ortiz Granja (2015) indica que, si la persona que enseña parte de la idea de que es poseedor del conocimiento que va a transmitir a los estudiantes, probablemente usará metodologías tradicionales que implican un proceso pasivo de aprendizaje, con los estudiantes en la postura de receptores del conocimiento. En cambio, si la persona que enseña parte del principio de que el conocimiento se construye, va a promover la participación activa de los estudiantes, va a entrar en diálogo con ellos, para lograr un ambiente de colaboración, en el cual es posible llegar a la construcción del conocimiento, tomando como base el acervo científico y tecnológico, acumulado por el ser humano a lo largo de su historia.

Como afirma García (2017), de esas diferentes formas de enseñar y aprender soportadas en sistemas digitales, van llegando a todos los rincones del planeta presentando distintos formatos según visiones pedagógicas, filosóficas, psicológicas, sociológicas y políticas, en función de las disponibilidades económicas y de los aportes tecnológicos. En efecto, como en otros ámbitos de la vida, son las tecnologías las que propician las disrupciones, también en la educación. En el caso que nos ocupa, los formatos educativos no presenciales se van imponiendo, en ocasiones, de forma brusca (disrupción), rompiendo espacios, tiempos, métodos, recursos, roles, etc., de sistemas educativos clásicos, estáticos, entumecidos, rígidos y con sólidas estructuras.

Por otra parte, las videoconferencias tradicionales están muy asociadas con los libros de texto, donde se limita al plan de estudios y la utilización de tecnología para facilitar las prácticas de enseñanza. De esta manera, las aulas centradas en el profesor tienden a tener niveles más bajos 
de participación de los estudiantes y un menor entusiasmo en una experiencia de aprendizaje activo. Evidentemente, cuando los estudiantes prestan menos atención durante las clases magistrales, puede causar aburrimiento, inquietud e incluso provocar comportamientos disruptivos innecesarios entre los compañeros de curso (Perera et al., 2020).

Principalmente en la educación virtual, las tecnologías digitales han saltado en un papel notable en la conformación y evolución de la sociedad de la información y de la sociedad del conocimiento. Por tanto, han impactado facultativamente en todos los espacios de la actividad humana, convirtiendo nuestras costumbres en la manera de cómo interactuamos, nos comunicamos y, por consiguiente, también la forma en que aprendemos en la vida diaria (Souto-Seijo et al., 2021). Es por ello que se debe innovar en la educación con los procesos de enseñanza y aprendizaje, en formar a estudiantes con perfiles disruptivos que solventen las necesitas de la sociedad y los problemas del país de crecer en el desarrollo y la innovación.

Según Mora \& Hooper (2016), pese a que el ambiente virtual permite múltiples espacios de comunicación para la realización del trabajo colaborativo, el hecho de no contar con la presencialidad resulta en que muchas veces los miembros del grupo no se puedan poner de acuerdo fácilmente en la dinámica o distribución del trabajo. Muchas veces también influye la experiencia previa que tengan en ambientes virtuales, pues el hecho de contar con experiencia facilita la comprensión de las dinámicas, así mismo, las competencias tecnológicas de cada quien influyen en la destreza que se tenga para el uso de la herramienta que se proponga para la colaboración, en este caso los Google Docs, que permiten compartir en tiempo real documentos y plantear ejercicios dando una mejor retroalimentación del contenido.

En cuanto en la educación online, una de las aplicaciones más utilizadas por los profesores de incluir gamificación es Quizizz, una App gratuita y también con su opción de pago, que permite al docente planificar varias actividades en línea como de tipo lección o evaluación; es decir, que puede ser realizada por los estudiantes en tiempo real, manipulando sus dispositivos electrónicos como: teléfonos inteligentes, tabletas o computadoras. También permite al docente autoevaluarse en el contenido impartido si está generando aprendizaje significativo indagando en los resultados y de reforzar ciertos aspectos que necesitan mejoras.

Teniendo en cuenta a Nurhayati (2020), a diferencia de otras aplicaciones educativas, los juegos educativos Quizizz tienen temas de personajes, memes, avatares y música que pueden entretener a los estudiantes durante el proceso de aprendizaje, practicar o hacer pruebas de forma independiente. Por tanto, el juego educativo Quizizz también permite a los estudiantes competir entre sí para alentarlos a ser más activos en el proceso de aprendizaje y motivados para hacer ejercicios y pruebas, con la esperanza de poder obtener resultados altos en las pruebas.

Teniendo en cuenta a Green \& Green (2018), Flipgrid es una plataforma de video discusión, diseñada para permitir que los estudiantes aborden temas innovadores para participar rápidamente en conversaciones grabadas de tipo video lecciones. Sin embargo, Flipgrid es una multiplataforma basada en la web y aplicación móvil. Hay dos términos clave en Flipgrid: cuadrícula y tema: 
una cuadrícula es lo que crea para una clase o curso, esta contiene los temas (discusiones) que crea para una clase o curso. Dentro de un tema existe la opción de tener varios alumnos que den respuestas rápidas o consultar dudas al profesor. Para Ruelas (2019), la plataforma Flipgrid va más allá de ser útil, pero está vinculada a la reflexividad y retención. Las interacciones sociales que se pueden crear en nombre del profesor son a través del uso de la tecnología más significativas. De este modo, el instructor tiene la oportunidad de crear un óptimo ambiente reflexivo en el desarrollo de pensamiento crítico.

A modo de concluir, las universidades reconocen la importancia de la educación virtual, aún no se han buscado mecanismos institucionales, pedagógicos y tecnológicos adecuados (Acevedo, 2015). Con el uso de la tecnología en la educación superior, existe la necesidad de comprender las prácticas activas de los estudiantes y profesores, con miras a repensar los enfoques del aprendizaje mejorado por la tecnología (Flavin, 2017). Las prácticas a distancia van progresivamente arrebatando espacio y tiempo a las formas más convencionales de enseñar y aprender (García, 2017). La innovación disruptiva es una teoría útil para comprender por qué algunas tecnologías tienen éxito en la educación superior y otras fracasan (Flavin, 2021). Por otra parte, el docente debe desaprender en el uso de tecnologías y volver a aprender en la innovación disruptiva, con nuevas herramientas digitales que activen al estudiante en su proceso de enseñanza y aprendizaje.

\section{Metodología}

La investigación se llevó a cabo en el contexto del proceso de enseñanza y aprendizaje en la Universidad Técnica de Manabí, Ecuador, de las carreras en línea. Por ende, se desarrolló un pilotaje a los estudiantes de primer nivel en la carrera de psicología modalidad en línea, que tiene mayor auge, con una población de 320 alumnos en el periodo académico noviembre del 2020 a abril del 2021.

El estudio parte de una intencionalidad exploratoria de conocer a fondo el problema en el proceso de enseñanza y aprendizaje en la práctica educativa en línea, para esto se procedió a indagar en una revisión literaria, de lo que se había investigado y lo que falta por investigar. El enfoque utilizado en esta investigación fue de corte cuantitativo con un diseño no experimental y transversal, de alcance descriptivo (Pérez et al., 2019), realizando un estudio del fenómeno comparado con otras asignaturas empleando tecnologías disruptivas en las clases sincrónicas.

En la población se realizó el tipo de muestreo no probabilístico aleatorio simple, de este modo se realizó una fórmula para obtener la muestra poblacional en los alumnos de la carrera de psicología en línea, donde esta se conformó con 136 estudiantes encuestados con un nivel confianza del $95 \%$ y un margen error $6.4 \%$. En cuanto al por qué de no tomar el total de la población de los estudiantes del primer nivel de psicología que son 320 educandos, dado que, no todos respondieron, por temor a represalias de los docentes, en el cual evaluaron el proceso de enseñanza-aprendizaje en la práctica en línea.

El instrumento que se utilizó para levantar la información fue la encuesta, cuyo principal objetivo fue medir el impacto que tiene el uso de las tecnologías disruptivas en el proceso de enseñanza y 
aprendizaje de las carreras en línea de la UTM, el tipo de cuestionario se ejecutó con 6 preguntas en la escala Likert, el cual se desarrolló en la aplicación de Google Forms. Una vez culminado se procedió a realizar la validación por expertos.

Por otra parte, la ejecución del instrumento se llevó a cabo en los meses de marzo y abril del 2021 , donde se procedió a enviarlo a los estudiantes por enlace compartido mediante la aplicación de Whatsapp en los grupos de la carrera de psicología modalidad en línea del primer semestre. Adicionalmente, se aplicó la observación participante mediante las videoconferencias sincrónicas de las carreras de psicología en línea, para confrontar los resultados de las encuestas. Se precisan limitaciones de que algunos estudiantes no realizaron la encuesta por temor represalias de los docentes al momento de evaluarlos.

\section{Resultados}

Se realizó un análisis sobre el impacto que causa al implementar tecnologías disruptivas en los alumnos en las clases sincrónicas, dado que, el docente inserta plataformas colaborativas para realizar prácticas en línea guiando y proponiendo debates a los estudiantes.

La primera pregunta que se realizó fue de carácter evaluativo para explorar, según su criterio, la calidad de las clases sincrónicas o asincrónicas con el docente tutor en el aprendizaje línea. Por ende, permitió conocer el grado de satisfacción del estudiante de que los profesores deben mejorar la metodología tradicional al momento de impartir la pedagogía en línea, de esta forma, en los cuadros se procede el anonimato del estudio comparativo de las asignaturas. Cabe recalcar que la asignatura 1 y 4 realizaron en sus procesos de enseñanza y aprendizaje la implementación de tecnologías disruptivas con plataformas colaborativas.

Tabla 1.

Encuesta a estudiantes sobre la calidad de las clases sincrónicas o asincrónicas

\begin{tabular}{lllll}
\hline \multirow{2}{*}{ Materia } & \multicolumn{4}{l}{$\begin{array}{l}\text { Pregunta 1. Evalúe según su criterio la calidad de las clases sincrónicas o } \\
\text { asincrónicas con el docente tutor en el aprendizaje línea. }\end{array}$} \\
\cline { 2 - 5 } & $\begin{array}{l}\text { Nada } \\
\text { satisfactorio }\end{array}$ & $\begin{array}{l}\text { Poco } \\
\text { satisfactorio }\end{array}$ & Muy satisfactorio & $\begin{array}{l}\text { Totalmente } \\
\text { satisfactorio }\end{array}$ \\
\hline Asignatura 1 & $5 \%$ & $4 \%$ & $47 \%$ & $80 \%$ \\
\hline Asignatura 2 & $12 \%$ & $28 \%$ & $52 \%$ & $44 \%$ \\
\hline Asignatura 3 & $5 \%$ & $6 \%$ & $60 \%$ & $65 \%$ \\
\hline Asignatura 4 & $5 \%$ & $5 \%$ & $48 \%$ & $78 \%$ \\
\hline Asignatura 5 & $5 \%$ & $11 \%$ & $56 \%$ & $64 \%$ \\
\hline
\end{tabular}

De acuerdo con la Tabla 1, los alumnos manifestaron que en las clases sincrónicas o asincrónicas, los docentes tutores de la asignatura 2, 3 y 5 deben mejorar la interacción y cambiar el modelo tradicional en la educación en línea, dado que, los mejores puntuados de la asignatura 1 y 4 utilizan tecnologías disruptivas con metodologías activas y hacen uso de videos, plataformas, software interactivo u otros recursos didácticos en línea, que permitan interacción y participación entre estudiante-docente. Por tanto, es muy importante fortalecer de las clases sincrónicas en 
línea, implementando tecnologías disruptivas y metodologías activas fomentando la interacción a tiempo real.

Concretamente, se consideró que el uso de las tecnologías digitales sólo tienen sentido si su utilización va más allá de la de servir de herramientas de sustitución y ampliación de otras tecnologías, y si posibilitan un rediseño significativo de las actividades de aprendizaje, para crear nuevas actividades de aprendizaje inconcebibles con otras herramientas; es decir, se busca entender las tecnologías digitales no solo como herramientas para la mejora sino para la transformación (Perera et al., 2020).

Tabla 2.

Encuesta a estudiantes sobre uso del docente de videos, plataformas, software interactivo u otros recursos didácticos

\begin{tabular}{lllll}
\hline \multirow{2}{*}{ Materia } & \multicolumn{4}{l}{$\begin{array}{l}\text { Pregunta 2. Con qué frecuencia en las clases sincrónicas o asincrónicas los } \\
\text { docentes tutores hacen uso de: videos, plataformas, software interactivo u } \\
\text { otros recursos didácticos online que permitan interacción o participación } \\
\text { entre compañeros }\end{array}$} \\
\cline { 2 - 5 } & Nada & Poco & Eventualmente & Frecuentemente \\
\hline Asignatura 1 & $0 \%$ & $6 \%$ & $30 \%$ & $100 \%$ \\
\hline Asignatura 2 & $17 \%$ & $36 \%$ & $50 \%$ & $33 \%$ \\
\hline Asignatura 3 & $7 \%$ & $15 \%$ & $56 \%$ & $58 \%$ \\
\hline Asignatura 4 & $4 \%$ & $11 \%$ & $37 \%$ & $84 \%$ \\
\hline Asignatura 5 & $8 \%$ & $21 \%$ & $49 \%$ & $58 \%$ \\
\hline
\end{tabular}

En la segunda pregunta se indagó para descubrir si los docentes utilizan algún recurso tecnológico para interactuar en las clases en línea, donde los informantes indicaron que en las clases sincrónicas o asincrónicas los profesores tutores no todos hacen uso de videos interactivos, plataformas colaborativas, o aplicaciones digitales, u otros recursos didácticos online que permitan fomentar interacción o participación entre sus compañeros y docente, tal como se evidencia en la Tabla 2.

De acuerdo con los estudios de Flavin \& Quintero (2018), iniciar desde cero en las estrategias de aprendizaje mediadas por la tecnología, puede conducir a la acomodación de la innovación disruptiva en la educación superior, dado que, evita un desajuste entre los enfoques tecnológicos articulados a través de estrategias en la práctica en línea, utilizando la tecnología como medio para fomentar la interacción en tiempo real con los estudiantes y profesores. Si bien se puede argumentar que las instituciones no deberían intentar innovación disruptiva, porque ocurre desde cero a través de un proceso de puesta en práctica, uno de los argumentos centrales es que las tecnologías disruptivas desembocan tecnologías establecidas, por lo tanto, es una buena idea que las universidades al menos estén al tanto de estas, porque de lo contrario, sus estrategias corren el riesgo de estar moribundas por las prácticas del día a día de estudiantes y profesores (pp. 2-3). 
Tabla 3.

Encuesta a estudiantes sobre qué herramientas digitales utiliza cada uno de los profesores

\begin{tabular}{llllll}
\hline Materia & \multicolumn{4}{l}{$\begin{array}{l}\text { Pregunta 3. Indique las herramientas digitales que utilizan cada uno de los } \\
\text { docentes para la impartición del contenido de las diferentes asignaturas. (Deje en } \\
\text { blanco si no utiliza esas herramientas }\end{array}$} \\
\cline { 2 - 6 } & $\begin{array}{l}\text { Google y sus } \\
\text { herramientas }\end{array}$ & Quizizz & Pizarras digitales & Kahoot & Flipgrid \\
\hline Asignatura 1 & $96 \%$ & $25 \%$ & $15 \%$ & $10 \%$ & $5 \%$ \\
\hline Asignatura 2 & $100 \%$ & $0 \%$ & $11 \%$ & $0 \%$ & $0 \%$ \\
\hline Asignatura 3 & $100 \%$ & $0 \%$ & $10 \%$ & $0 \%$ & $0 \%$ \\
\hline Asignatura 4 & $100 \%$ & $0 \%$ & $14 \%$ & $5 \%$ & $0 \%$ \\
\hline Asignatura 5 & $100 \%$ & $0 \%$ & $14 \%$ & $0 \%$ & $0 \%$ \\
\hline
\end{tabular}

La tercera pregunta se realizó de carácter descriptiva, sobre qué herramientas digitales utilizan los profesores en la participación de contenidos. Los encuestados mencionaron que no todos los docentes utilizan tecnologías en sus clases, de vez en cuando para realizar una tutoría. En cuanto a la observación participante, manifestaron que algunos profesores son monótonos al leer el contenido a pie de la letra, tal como está en el compendio y con la cámara apagada. Sin embargo, hay algunos educadores que, si van explicando paso a paso, realizando pausas en la clase en línea, de este modo para preguntar si está clara la explicación y da la opción de repetir, por otra parte, algunos pedagogos realizan prácticas en la videoconferencia a tiempo real, acordes al contenido desarrollado, aunque no todos realizan interacción con aplicaciones virtuales de tipo lección con Quizizz o Kahoot, que se divierten resolviendo preguntas.

Este resultado es coincidente con lo planteado por Gaete-Quezada (2011), cuando sugiere que el personal docente utilice estrategias didácticas e innovadoras para el logro de aprendizaje significativo, que induzcan a potenciar un ejercicio de una docencia más constructivista que tendrá impacto en el estudiantado.

Tabla 4.

Encuestas a estudiantes sobre la calidad de las herramientas utilizadas por el profesor

\begin{tabular}{lllll}
\hline \multirow{2}{*}{ Materia } & \multicolumn{4}{l}{$\begin{array}{l}\text { Pregunta 4. Evalúe según su criterio la calidad de las herramientas utilizadas } \\
\text { por el profesor en actividades tales como presentaciones, ejercicios, } \\
\text { actividades prácticas, videos. }\end{array}$} \\
\cline { 2 - 5 } & $\begin{array}{l}\text { Nada } \\
\text { satisfactorio }\end{array}$ & $\begin{array}{l}\text { Poco } \\
\text { satisfactorio }\end{array}$ & Muy satisfactorio & $\begin{array}{l}\text { Totalmente } \\
\text { satisfactorio }\end{array}$ \\
\hline Asignatura 1 & $2 \%$ & $6 \%$ & $50 \%$ & $78 \%$ \\
\hline Asignatura 2 & $11 \%$ & $31 \%$ & $70 \%$ & $24 \%$ \\
\hline Asignatura 3 & $2 \%$ & $13 \%$ & $72 \%$ & $49 \%$ \\
\hline Asignatura 4 & $2 \%$ & $11 \%$ & $58 \%$ & $65 \%$ \\
\hline Asignatura 5 & $2 \%$ & $15 \%$ & $71 \%$ & $48 \%$ \\
\hline
\end{tabular}

En cuanto a la cuarta pregunta, esta fue de conocimiento sobre las herramientas empleadas en las clases sincrónicas, si los profesores realizaban ejercicios en clase o proponían alguna actividad 
práctica. Por tanto, los participantes coinciden que la evaluación de los criterios de la calidad de las herramientas utilizadas por el profesor en actividades tales como presentaciones, ejercicios, actividades prácticas, videos. Algunos contenidos en las presentaciones están claros; sin embargo, faltaría reforzar con ejercicios prácticos o actividades interactivas utilizando aplicaciones virtuales para compartir con los compañeros.

Las clases en línea o cursos masivos como los MOOC que adoptan una escala e intensidad adecuadas, potencialmente y modifican la educación tradicional con nuevos modelos de entrega de educación superior, ampliando el acceso a una educación de calidad, lo que brinda oportunidades para un aprendizaje enriquecido. Experiencias con una comunidad global en línea y educadores consumados a través de la red y que los estudiantes aprendan a su propio ritmo adaptando sus estudios a su horario preferido volver a ver partes de las video conferencias (Rambe \& Moeti, 2016).

\section{Figura 1.}

Encuestas a estudiantes sobre su decisión en relación al uso de plataformas interactivas

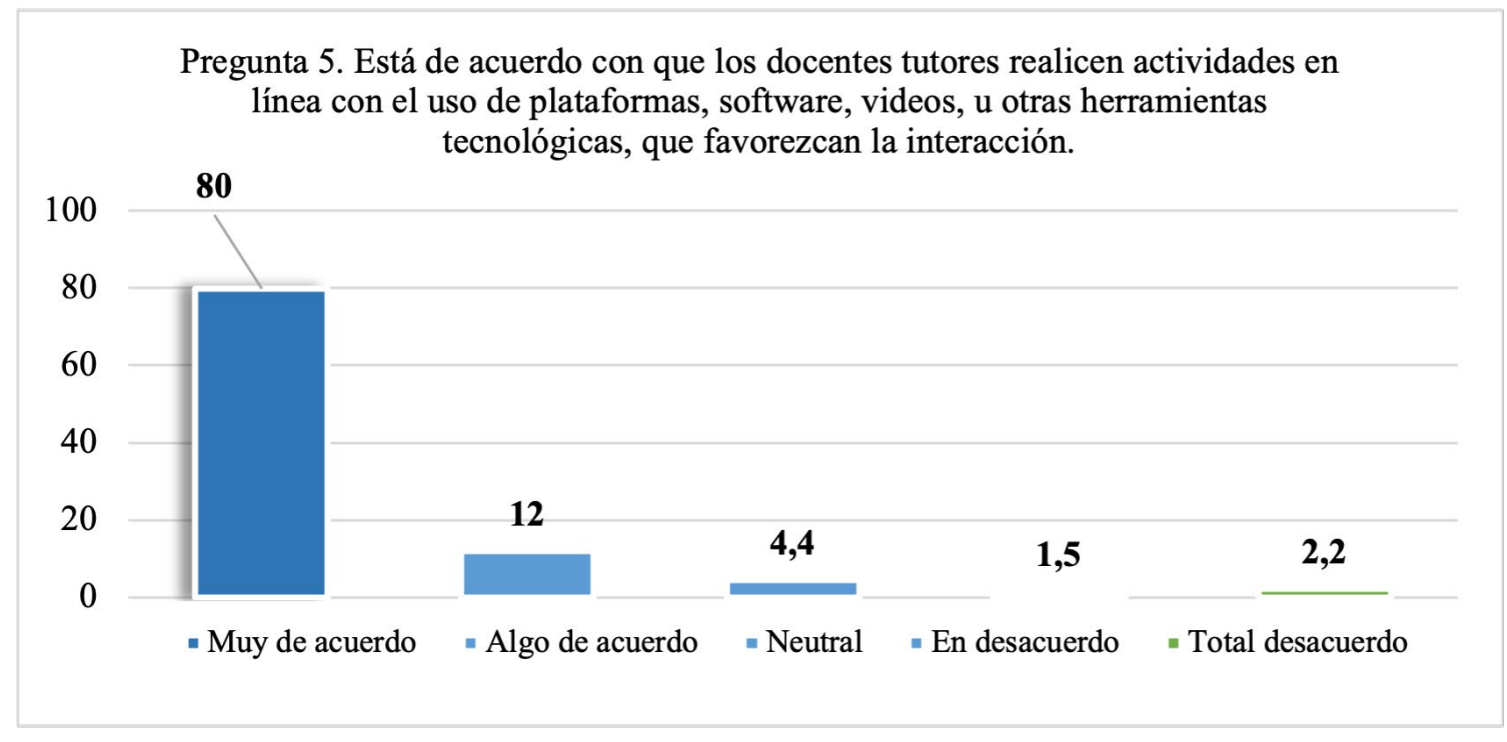

Como se observa en la Figura 1, los alumnos evaluaron que están de acuerdo con el $80 \%$ en que se debe implementar tecnologías disruptivas con plataformas colaborativas, permitiendo realizar prácticas a tiempo real, que permitan interactuar con el docente resolviendo inquietudes mejorando el proceso de enseñanza aprendizaje.

Estás innovaciones disruptivas rompen los paradigmas anteriores y producen drásticos cambios en la forma de hacer las cosas (Pérez et al., 2019). Implementar tecnologías como medio para realizar prácticas en línea es de mucha ayuda en el docente, de esta manera, discrepan algunas inquietudes del alumno surgida en clase en línea, permitiendo retroalimentar cada parámetro del ejercicio o actividad. 
Figura 2.

Encuestas a estudiantes sobre comparación en la implementación de tecnologías disruptivas

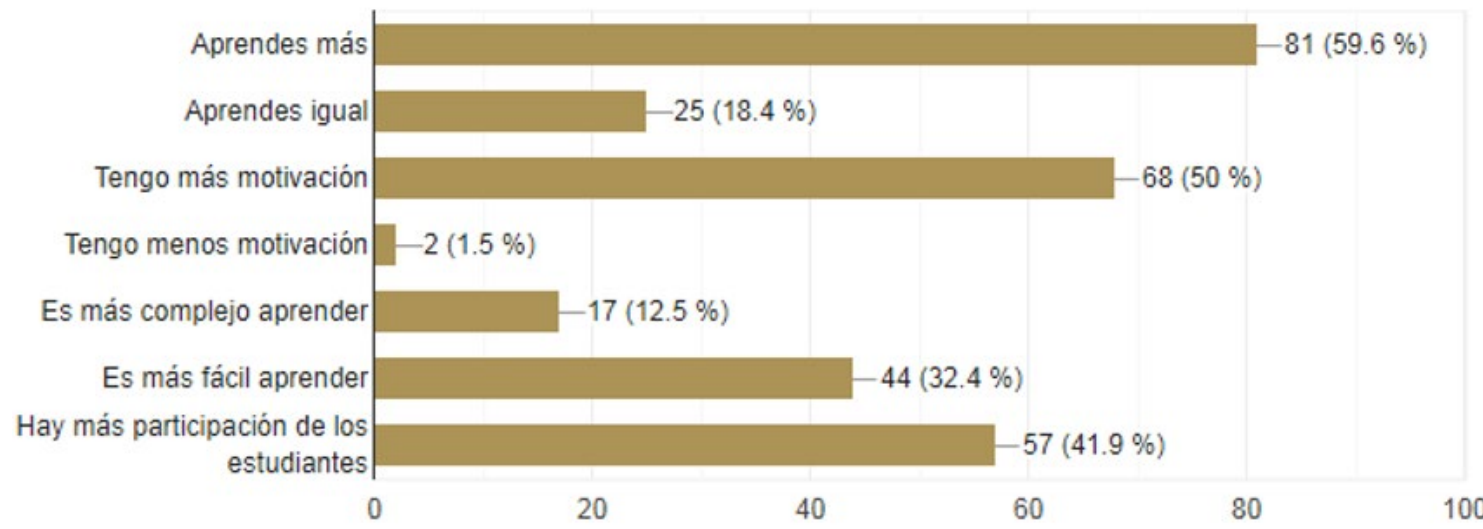

Para finalizar, la sexta pregunta se realizó en base al estudio comparativo de las dos asignaturas que implementaron tecnologías disruptivas, donde los estudiantes indican que las clases deben adquirir una buena combinación en los fundamentos teóricos y con las prácticas en línea, dado que, el profesor tutor debe planificar y diseñar nuevas estrategias, insertando herramientas tecnológicas con metodologías activas, como medio para interactuar en el aula virtual. De este modo, los alumnos pueden realizar a tiempo real los ejercicios o revisarlos después en los videos asincrónicos, el cual le permite reforzar sus conocimientos con el seguimiento de las prácticas, como se muestra en la Figura 2.

\section{Discusión}

En la investigación se exploraron los problemas y dio a conocer el impacto de las tecnologías disruptivas como medio de interacción en la participación activa de las clases sincrónicas, a manera de formular buenos entornos de educación superior, donde se observan dos asignaturas que sobresalen en el cambio del proceso enseñanza y aprendizaje implementando tecnologías disruptivas, para fortalecer la interacción, el aprendizaje colaborativo en línea y las prácticas a tiempo real, por ende, el estudiante recibe una retroalimentación clara en caso de que esté realizado mal el ejercicio o actividad. Sin embargo, al implementar tecnologías disruptivas en el aula, al principio puede ser confuso por iniciar desde cero, se debe tener claro las actividades a realizar y adaptar a la planificación curricular.

Por otra parte, planificar actividades es un reto al momento de implementar, se debe tener presente el objetivo que se desea que alcancen los estudiantes, al momento de combinar tecnologías disruptivas y metodologías activas de aprendizaje, aplicando un método de caso o de resolver ejercicios.

Como menciona Perera et al. (2020), con el uso de herramientas tecnológicas, este programa combinado mejoró la participación de los estudiantes en las actividades interactivas de la clase. Estas estrategias combinadas generaron una experiencia de aprendizaje significativa para 
todos los estudiantes, lo que les permitió participar plenamente en las discusiones de clase y presentaciones grupales. Los estudiantes respondieron positivamente sobre sus sesiones de clase interactivas.

Por otra parte, la investigación sugiere que las herramientas de tecnología de aprendizaje gratuito como los Google Docs, Quizizz y Flipgrid son tecnologías disruptivas de aprendizaje útiles en su adaptación para fomentar la interacción, la participación y la retención del aprendizaje en la educación online. Por ende, los profesores pueden utilizar estas herramientas alternativamente para albergar la interacción de preguntas inmediata en sus clases, luego se podría realizar una actividad de evaluación entre pares para alentar a los estudiantes a leer y aprender de estos. Las herramientas colaborativas de aprendizaje de Google, como documentos de hoja en blanco, hojas de cálculo y los formularios de Google, podrían aplicarse a los cursos para realizar evaluaciones y resolver problemas o de guiar el aprendizaje basado en proyectos.

La interacción entre profesores y estudiantes, y entre compañeros, es importante dado que la interacción promueve el aprendizaje activo, y es más probable que los estudiantes activos internalicen el conocimiento y logren una capacidad de aprendizaje de alto nivel. Las herramientas de integración con la tecnología de aprendizaje en clase se clasifican según la dimensión de conocimiento de aprendizaje específico (Wang, 2020). Es muy importante destacar que gran parte de los estudiantes está de acuerdo de que se inserten tecnologías disruptivas en las clases sincrónicas, realizando prácticas en línea y que ocurra la internación entre estudiante-docente.

Para culminar, los estudiantes mencionan que se sienten motivados y que aprenden más al momento de trabajar en clases sincrónicas, de que el profesor cambia su roll en guiar al alumno con parámetros claros en realizar prácticas a tiempo real, por ende, también se interesa en resolver dudas acerca del ejercicio o proyecto en ejecución, permitiendo al estudiante ser el centro de su propio estudio, en resolver problemas y de generar debates estando activo en clase.

\section{Conclusiones}

A modo de concluir, se muestran hallazgos positivos que genera el impacto de las tecnologías disruptivas, donde se obtuvieron grandes mejoras en el proceso pedagógico al aplicarlas y trabajar con ellas, fomentando la innovación dentro del aula. Asimismo, nos indican la riqueza en las interacciones y la posibilidad que tiene el estudiante de construir nuevas ideas de forma activa, siendo consciente de su propio conocimiento del aprendizaje en línea.

En parte del estudio exploratorio intencional se pudo observar que a los estudiantes no se les dificulta aprender ciertos temas, pero les gustaría que sus clases fueran más dinámicas; es decir, que muestren mayor interés cuando se utiliza las tecnologías disruptivas en dar solución a problemas, de esta manera se obtiene mejores resultados. Por otra parte, en cuanto a los docentes, algunos están de acuerdo en que se utilicen recursos didácticos digitales para mejorar la enseñanza en línea, que está basada en la innovación y su despliegue de nuevas metodologías activas. 
Considerando los beneficios que se pueden obtener en mejorar el proceso de enseñanza y aprendizaje, con estrategias pedagógicas, aplicando tecnologías disruptivas con plataformas virtuales de aprendizaje como medio para interactuar con el estudiante y fortalecer el pensamiento crítico, como trabajo futuro, es necesario seguir investigando en relación al tipo de interacciones que surgen al trabajar con herramienta actuales, dado que en un estudio más detallado se podrá entender la dinámica que se genera al aprender con este tipo de mecanismos y brindar a la evaluación formativa por parte del docente. 


\section{Referencias}

Acevedo, Á. (2015). Nuevas enseñanzas disruptivas en la educación superior en ciencias sociales. Los cursos en línea masivos y abiertos (MOOCs). Revista TEMAS, 3(9), 125-136. https://doi.org/10.15332/rt. v3i9.1366

Arriassecq, I., \& Santos, G. (2017). Nuevas tecnologías de la información como facilitadoras de Aprendizaje significativo. Archivos de Ciencias de la Educación, 11(12). https://doi.org/10.24215/23468866e030

Banoy, W. (2019). El uso pedagógico de las tecnologías de la información y la comunicación (TIC) y su influencia en el aprendizaje significativo de estudiantes de media técnica en Zipaquirá, Colombia. Academia y Virtualidad, 12(2), 23-46. https://doi.org/10.18359/ravi.4007

Cabecero, J. (2010). Los retos de la integración de las TICs en los procesos educativos. Límites y posibilidades. Perspectiva Educacional, formación de profesores, 49(1), 32-61. https://www.redalyc.org/ pdf/3333/333327288002.pdf

Christensen, C., \& Armstrong, E. (1998). Disruptive technologies: A credible threat to leading programs in continuing medical education? The Journal of Continuing in the Health Professions, 18(2), 69-80. https://doi. org/10.1002/chp.1340180202

Diaz-leante, I. (2019). Economía colaborativa e innovación disruptiva: Ios casos de Airbnb y Uber. [Tesis de grado, Universidad Pontificia Comillas]. http://hdl.handle.net/11531/32349

Flavin, M. (2012). Disruptive technologies in higher education. Research in Learning Technology, 20, $102-112$. https://doi.org/10.3402/rlt.v20i0.19184

Flavin, M. (2016). Disruptive conduct: the impact of disruptive technologies on social relations in higher education. Innovations in Education and Teaching International, 53(1), 1-13. https://doi.org/10.1080/14703297. $\underline{2013.866330}$

Flavin, M. (2017). Disruptive technology enhanced learning: The use and misuse of digital technologies in higher education. Springer. https://doi.org/DOI 10.1057/978-1-137-57284-4

Flavin, M. (2021). A Disruptive Innovation perspective on students' opinions of online assessment. Research in Learning Technology, 29, 1-14. https://doi.org/10.25304/rlt.v29.2611

Flavin, M., \& Quintero, V. (2018). UK higher education institutions' technology-enhanced learning strategies from the perspective of disruptive innovation. Research in Learning Technology, 26, 1-13. https://doi. org/10.25304/rlt.v26.1987 
Gaete-Quezada, R. A. (2011). El juego de roles como estrategia de evaluación de aprendizajes universitarios. Educación y Educadores, 14(2), 289-307. https://doi.org/10.5294/edu.2011.14.2.3

Gallardo, I., Castro, A., \& Saiz, H. (2020). Interacción y uso de tecnologías en los procesos de enseñanza y aprendizaje. Educatio Siglo XXI, 38(1), 119-138. https://doi.org/10.6018/educatio.413441

García, L. (2017). Educación a distancia y virtual: calidad, disrupción, aprendizajes adaptativo y móvil. RIED. Revista Iberoamericana De Educación a Distancia, 20(2), 9-25. https://doi.org/10.5944/ried.20.2.18737

Green, T., \& Green, J. (2018). Flipgrid: Adding Voice and Video to Online Discussions. TechTrends, 62, $128-130$. https://doi.org/10.1007/s11528-017-0241-x

Hutchings, M., \& Quinney, A. (2015). The Flipped Classroom, Disruptive Pedagogies, Enabling Technologies and Wicked Problems: Responding to" The Bomb in the Basement". Electronic Journal of e-learning, 13(2), 106-119. https://eric.ed.gov/?id=EJ1060159

Kurtz, H., Lloyd, S., \& Harwin, A. (2019). Teachers and Ed-Tech Innovation: Results of a National Survey. Editorial Projects in Education.

McAuley, A., Stewart, B., Siemens, G., \& Cormier, D. (2010). The MOOC model for digital practice.

Mora-Vicarioli, F., \& Hooper-Simpson, C. (2016). Collaborative Work in Virtual Learning Environments: Some Reflections and Prospects of Students. Revista Electrónica Educare, 20(2), 1-26. https://doi.org/10.15359/ ree.20-2.19

Nurhayati, E. (2020). Meningkatkan keaktifan siswa dalam pembelajaran daring melalui media game edukasi quiziz pada masa pencegahan penyebaran covid-19. Jurnal Paedagogy, 7(3), 145-150. https://doi. org/10.33394/jp.v7i3.2645

Ortiz Granja, D. (2015). El constructivismo como teoría y método de enseñanza. Sophia, (19), 93-110. https:// doi.org/10.17163/soph.n19.2015.04

Perera, C. J., Zainuddin, Z., Piaw, C. Y., Cheah, K. S. L., \& Asirvatham, D. (2020). The Pedagogical Frontiers of Urban Higher Education: Blended Learning and Co-Lecturing. Education and Urban Society, 52(9), 1305-1329. https://doi.org/10.1177/0013124519894966

Pérez, K., Rodríguez, I., \& Luna, S. (2019). Nivel de conocimiento de las Tecnologías Disruptivas en Educación Superior en estudiantes de la Universidad Autónoma de Nuevo León. Vincula Tégica, 527-536.

Pilonieta, G. (2017). Innovación disruptiva. Esperanza para la educación de futuro. Revista Educación y Ciudad, (32), 53-63. https://doi.org/10.36737/01230425.v0.n32.2017.1627 
Rambe, P., \& Moeti, M. (2016). Disrupting and democratising higher education provision or entrenching academic elitism: towards a model of MOOCs adoption at African universities. Educational Technology Research and Development, 65(3), 631-651. https://doi.org/10.1007/s11423-016-9500-3

Ruelas, D. M. (2019). Enhancing Online Learning for Public Health Graduate Students. Journal of Instructional Research, 8(2), 97-101. https://doi.org/10.9743/JIR.2019.8.2.12

Souto-Seijo, A., Estévez, I., \& Sande, O. (2021). Oportunidades de aprendizaje y formación docente: una mirada desde las Ecologías de Aprendizaje. Educatio Siglo XXI, 39(2), 61-80. https://doi.org/10.6018/educa$\underline{\text { tio.463211 }}$

Wang, Y. (2020). Design-based research on integrating learning technology tools into higher education classes to achieve active learning. Computers \& Education, 156. https://doi.org/10.1016/j.compedu.2020.103935 
Copyright (c) 2022 Walter José Zambrano Romero y Jaime Alcides Meza Hormaza

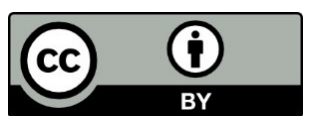

Este texto está protegido bajo una licencia internacional Creative Commons 4.0.

Usted es libre para Compartir-copiar y redistribuir el material en cualquier medio o formato

- y Adaptar el documento - remezclar, transformar y crear a partir del material-para cualquier propósito, incluso para fines comerciales, siempre que cumpla las condiciones de Atribución. Usted debe dar crédito a la obra original de manera adecuada, proporcionar un enlace a la licencia, e indicar si se han realizado cambios. Puede hacerlo en cualquier forma razonable, pero no de forma tal que sugiera que tiene el apoyo del licenciante o lo recibe por el uso que hace de la obra.

\section{Resumen de licencia - Texto completo de la licencia}

